\section{(2) \\ BRAZILIIAN JOURNAL \\ OF MEDICAL AND BIOLOGICAL RESEARCH}

www.bjournal.com.br
ISSN 0100-879X

Volume 45 (3) 179-290 March 2012

BIOMIEDICAL SCIENCES

AND

CLINICAL INVESTIGATION

Braz J Med Biol Res, March 2012, Volume 45(3) 230-237

doi: 10.1590/S0100-879X2012007500009

A conjugate of an anti-midkine single-chain variable fragment to doxorubicin inhibits tumor growth

Shuli Zhao, Guangfeng Zhao1, Hao Xie1, Yahong Huang1 and Yayi Hou

The Brazilian Journal of Medical and Biological Research is partially financed by

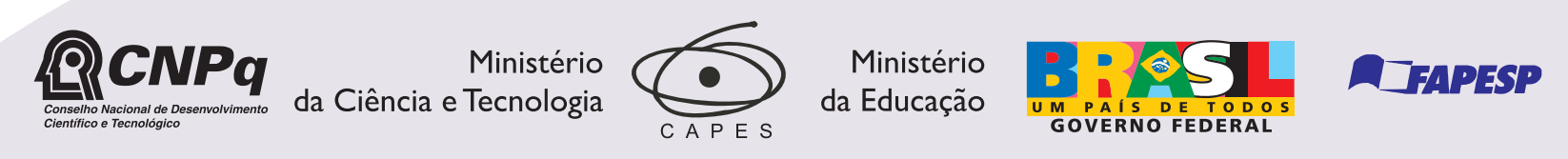

scie/
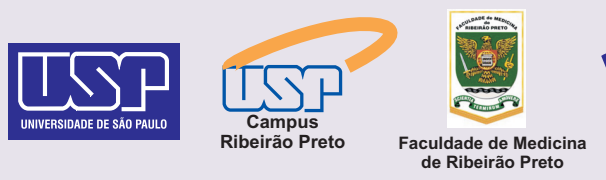

Institutional Sponsors

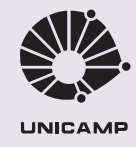

SHIMADZU

$\oplus$ UNICAMP

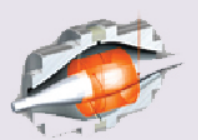

1EDD Associaçä Explore High - Performance MS Orbitrap Technology In Proteomics \& Metabolomics analítice $\underset{\text { analiticaweb.com.br }}{\text { Thermo }}$ S I T I I IC 


\title{
A conjugate of an anti-midkine single-chain variable fragment to doxorubicin inhibits tumor growth
}

\author{
Shuli Zhao ${ }^{1,2}$, Guangfeng Zhao ${ }^{1}$, Hao Xie ${ }^{1}$, Yahong Huang ${ }^{1}$ and Yayi Hou ${ }^{1,3}$ \\ ${ }^{1}$ Immunology and Reproductive Biology Laboratory, Medical School \& State Key \\ Laboratory of Pharmaceutical Biotechnology, Nanjing University, Nanjing, China \\ ${ }^{2}$ Nanjing Affiliated First Hospital, Nanjing Medical University, Nanjing, China \\ ${ }^{3}$ Jiangsu Key Laboratory of Molecular Medicine, Nanjing University, Nanjing, China
}

\begin{abstract}
Doxorubicin (DOX) was conjugated to a single-chain variable fragment (scFv) against human midkine (MK), and the conjugate (scFv-DOX) was used to target the chemotherapeutic agent to a mouse solid tumor model in which the tumor cells expressed high levels of human MK. The His-tagged recombinant scFv was expressed in bacteria, purified by metal affinity chromatography, and then conjugated to DOX using oxidative dextran (Dex) as a linker. The molecular formula of this immunoconjugate was scFv(Dex $)_{1.3}(\mathrm{DOX})_{20}$. In vitro apoptosis assays showed that the scFv-DOX conjugate was more cytotoxic against MK-transfected human adenocarcinoma cells (BGC823-MK) than untransfected cells $(55.3 \pm 2.4$ vs $22.4 \pm 3.8 \%)$ for three independent experiments. Nude mice bearing BGC823-MK solid tumors received scFv-DOX or equivalent doses of scFv + DOX for 2 weeks and tumor growth was more effectively inhibited by the scFv-DOX conjugate than by scFv + DOX (51.83\% inhibition vs $40.81 \%)$. Histological analysis of the tumor tissues revealed that the highest levels of DOX accumulated in tumors from mice treated with scFv-DOX and this resulted in more extensive tumor cell death than in animals treated with the equivalent dose of scFv + DOX. These results show that the scFv-DOX conjugate effectively inhibited tumor growth in vivo and suggest that antigen-specific scFv may be competent drug-carriers.
\end{abstract}

Key words: Single-chain variable fragments; Midkine; Tumor targeting; Doxorubicin

\section{Introduction}

Growth factors are often overexpressed by tumors, and many growth factors, receptors, and receptor-signaling components have become effective targets for cancer treatment (1). Midkine (MK) is a heparin-binding growth factor with a molecular mass of $13 \mathrm{kDa}$ that is expressed at high levels in many types of cancer $(2,3)$. MK has a number of biological activities and is involved in the transformation of fibroblasts, cell growth, cell survival, cell migration, and angiogenesis $(4,5)$. A previous study of Chinese patients by our group showed that MK is highly expressed in gastric cancer tissue. Furthermore, the expression levels of MK mRNA and protein correlated with the clinical stage and distant metastasis of gastric cancer $(6,7)$. Inhibition of MK expression by small interfering RNA had an antitumor effect on gastric cancer (8). Recently, it was reported that plasma MK levels were increased in patients with breast cancer and osteosarcoma $(9,10)$. Once the tumor-specific expression pattern of $\mathrm{MK}$ is demonstrated, it may be possible to target MK antigens to induce anti-tumor immune responses in vivo, or to target MK for the development of antibody-based therapeutics (11). Taken together, these data suggest that MK may contribute to oncogenesis and tumor progression, and may be useful as a cancer biomarker and/or a target for immunotherapy.

Many malignant tissues can be distinguished from their normal counterparts by the expression or overexpression of cell surface and intracellular molecules. In many cases, it is possible to target these antigens, particularly those that show overexpression on the cancer cell surface. Monoclonal antibodies (mAbs) with high binding specificity to tumorspecific antigens can perform this task effectively. Such $\mathrm{mAbs}$ have been used as vehicles to deliver cytotoxic drugs

Correspondence: Yayi Hou, Nanjing University, 22 Hankou Road, Nanjing, Jiangsu 210093, China. Fax: +86-25-8368-6441.

E-mail: yayihou@nju.edu.cn

Received April 10, 2011. Accepted January 13, 2012. Available online January 27, 2012. Published March 19, 2012. 
to specific tumor cells (12). However, the large molecular size of most mAb-drug immunoconjugates often results in poor penetration into solid tumors. Single-chain variable fragments (scFv) have been developed to overcome this problem. An scFv is a small, engineered fusion protein (30 kDa) composed of the variable heavy chain $(\mathrm{VH})$ and light chain $(\mathrm{VL})$ of an antibody connected by a short, flexible polypeptide linker (13). This antibody fragment retains the original antigen-binding site, thereby maintaining the same specificity and affinity for the antigen as the parent antibody (14). There are many advantages to the use of scFv, including the stability of the protein, the ability to produce large quantities in Escherichia coli at low cost, and the ability to improve the affinity for an antigen through genetic engineering to modify the amino acid sequence (15-17). The potential applications of scFv include in vivo radioimmunodiagnostic techniques and radiotherapy, and targeted delivery of drugs and toxins for the treatment of cancer and infections (18).

We have reported the successful cloning of the $\mathrm{VH}$ and VLgenes from the 9E4 hybridoma cell line, which secretes an MK-specific mAb. Formation of the scFv gene from the 9E4 $\mathrm{VH}$ and VL was accomplished by RT-PCR and insertion of $a$ (Gly4Ser)4 linker. This gene was then cloned into the bacterial expression vector $\mathrm{pET} 30 \mathrm{a}(+)$ and expressed in E. coli. The expressed and purified MK-specific scFv had the same affinity as the parent 9E4 mAb, and immunohistochemical analyses revealed positive staining in tissue from a patient with adenocarcinoma of the colon (19).

Here, we explored the possibility of using the anti-MK scFv conjugated to doxorubicin (DOX) as a targeted therapy for the treatment of cancer. DOX is a potent cytotoxic and antiproliferative drug and has been frequently used to produce antibody-toxin conjugates for cancer therapy (20).

\section{Material and Methods}

\section{Cell culture}

We previously developed the $9 \mathrm{E} 4$ hybridoma cell line that produces an mAb against human MK. The BGC823 cell line, a poorly differentiated human gastric adenocarcinoma cell line, was obtained from the Institute of Biochemistry and Cell Biology, Chinese Academy of Sciences. BGC823-MK cells, which were transfected with a pcDNA3.1/MK vector and which secrete human MK, were developed in our laboratory (7). All cell lines were cultured in RPMI 1640 medium (Gibco-BRL, USA) supplemented with $10 \%$ fetal bovine serum (Sijiqing Bio Corp., China) and $100 \mu \mathrm{g} / \mathrm{mL}$ each of streptomycin and penicillin $\mathrm{G}$ (Amresco, USA), at $37^{\circ} \mathrm{C}$ in a humidified $5 \%$ $\mathrm{CO}_{2}$ atmosphere.

\section{Expression and purification of scFv}

The development, expression, and purification of MKspecific-scFv (referred to here as scFv) were conducted as previously described (21). Briefly, E. coli BL21(DE3) cells containing the pET30a(+)-scFv-6X His-tag vector $(\mathrm{pET} 30 \mathrm{a}(+)$ from Novagen, USA) were grown at $37^{\circ} \mathrm{C}$ in Luria broth supplemented with $100 \mu \mathrm{g} / \mathrm{mL}$ kanamycin (Amresco). Protein expression was induced with $0.1 \mathrm{mM}$ isopropyl-1-thio- $\beta-D-$ galactopyranoside (IPTG; Sigma, USA). Following induction, the bacteria were harvested by centrifugation and were then sonicated (ten 20 -s pulses, with 2-s intervals between pulses) with an Ultrasonic Disrupter (Sanyo 150, Gene Company Limited, Japan) at $0^{\circ} \mathrm{C}$. The scFv-containing inclusion bodies were harvested by centrifugation of the sonicate and were solubilized overnight at room temperature in solubilizing buffer (50 mM sodium phosphate buffer, pH 8.0, 8.0 M urea, $1.0 \mathrm{mM}$ DTT, and 0.1 mM PMSF (Sigma). The scFv proteins were refolded by dialysis of the lysate at $4^{\circ} \mathrm{C}$. The dialysate was then centrifuged and the supernatant was applied to an immobilized metal affinity chromatography column (Chelating Sepharose 6B, $5 \mathrm{~mL}$ column volume; Pharmacia, Sweden) and the scFv was purified according to manufacturer specifications. The purity of the eluted scFv protein was determined by SDS-PAGE followed by staining with Coomassie brilliant blue G-250 (Merck, Germany).

\section{Preparation of ScFv-DOX conjugates}

The purified ScFv was coupled to DOX through the aldehyde groups of polyaldodextraven (PAD) as reported, with some modifications (22). Briefly, PAD was prepared by dissolving $1 \mathrm{~g}$ Dex-T10 (10 kDa dextran, Dex; Pharmacia) in $100 \mathrm{~mL} 0.1 \mathrm{M}$ sodium acetate, $\mathrm{pH} 5.5$, containing 0.03 $\mathrm{M} \mathrm{NalO}_{4}$, and incubated for $20 \mathrm{~h}$ at $4^{\circ} \mathrm{C}$. PAD (60 mg) was then added to $1 \mathrm{~mL}$ phosphate-buffered saline (PBS) containing DOX (18 mg) and the mixture was incubated for $48 \mathrm{~h}$ at $4^{\circ} \mathrm{C}$. Then, $10 \mathrm{mg}$ purified scFv was added and the incubation was continued for a further $48 \mathrm{~h}$ at $4^{\circ} \mathrm{C}$. Finally, sodium borohydride $(0.5 \mathrm{mg})$ was added to neutralize the free aldehyde groups. The conjugate was then purified from the mixture on a Sephadex G150 column. Protein analysis of the eluate with a Nucleoalbumin Detector (Model WXJ-9388, Sibas Biotechnology Development Co., Ltd., China) revealed two peaks, the first of which, a high molecular weight peak, was found to be the scFv-DOX conjugate. The peaks were collected and the protein purity was confirmed by SDS-PAGE with Coomassie brilliant blue staining.

\section{Analysis of scFv-DOX conjugates}

The purified scFv-DOX conjugates were analyzed for 1) scFv content using the BCA protein assay (Beyotime, China); 2) DOX content by comparison with a standard curve of DOX (Sigma) (23), and 3) Dex content by comparison with a standard curve, using the phenol-sulfuric acid assay for carbohydrates, as previously described (24).

\section{Affinity of scFv-DOX conjugates}

The affinity of the purified scFv-DOX conjugate for human MK (Peprotech, USA) was examined by ELISA (25). Briefly, a 96-well plate was coated with $100 \mathrm{ng} / \mathrm{mL}$ human MK. Wells were blocked with $5 \%$ bovine serum albumin (BSA) for $1 \mathrm{~h}$, 
and serial dilutions of the free ScFv or scFv-DOX conjugate were then added to the wells. Plates were incubated for $2 \mathrm{~h}$ at $37^{\circ} \mathrm{C}$ and then washed three times. A mouse anti-His antibody at 1:2000 dilution (Qiagen, USA) was then added, and the plate was incubated for $1 \mathrm{~h}$ at $37^{\circ} \mathrm{C}$. Wells were washed again, and a horseradish peroxidase (HRP)-conjugated secondary rabbit anti-mouse lgG was added to all wells. Finally, the HRP chromogen o-phenylenediamine was added and the plates were incubated at room temperature for $15 \mathrm{~min}$. Absorbance at $490 \mathrm{~nm}$ was measured with a Synergy HT multifunctional microplate reader (BioTek, USA).

\section{Cell binding of the scFv-DOX conjugate}

An immunofluorescence assay was used to determine the in vivo binding activity of scFv-DOX. In this assay, the scFv-DOX was used to detect MK expression in mesenchymal stem cells (MSCs, from the Stem Cell Center of Jiangsu Province, Beike Bio-Technology, China). MSCs grown on coverslips were washed and fixed with $4 \%$ paraformaldehyde, followed by washing with $0.2 \%$ Triton X-100. Coverslips were incubated with $5 \%$ BSA to reduce nonspecific binding. The coverslips were subsequently incubated at $4^{\circ} \mathrm{C}$ overnight with an scFv-DOX solution. A mouse anti-His antibody at 1:2000 dilution (Qiagen) was then added. After washing, rhodamineconjugated AffiniPure goat anti-mouse IgG was used as the secondary antibody (1:200, Santa Cruz, USA). Counterstaining was performed using $1 \mu \mathrm{g} / \mathrm{mL}$ 4',6-diamidino-2-phenylindole (DAPI, Sigma). A confocal microscope (Leica, Germany) was used to observe the stained cells and to detect the expression of $\mathrm{MK}$ to validate the in vivo binding of scFv-DOX.

\section{In vitro cytotoxicity assay}

The cytotoxicity of scFv-DOX was assessed in vitro by measuring apoptosis of BGC823 and BGC823-MK cells. Cells were cultured in 48-well plates at a density of $1 \times 10^{6}$ cells/well and treated with DOX, scFv, and scFv-DOX for 4 h. The final concentrations were $2.5 \mu \mathrm{g} / \mathrm{mL}$ or equivalent of DOX, and $4.375 \mu \mathrm{g} / \mathrm{mL}$ or equivalent of scFv. Apoptosis was measured by propidium iodide (PI) binding to DNA as described in a previous report (26).

\section{Animal tumor model}

Healthy female BALB/c nude mice (5-6 weeks old) were obtained from Vital River Laboratory Animal Co., Ltd., Beijing Laboratory Animal Research Center (Beijing, China) and were housed in specific pathogen-free conditions at Drum Tower Hospital Animal Center, Nanjing, China, in a room controlled for temperature $\left(21 \pm 2^{\circ} \mathrm{C}\right)$, humidity $(55 \pm 5 \%)$, and light (12-h light/dark cycle). Water was available to the mice ad libitum. All procedures involving animals were approved by the Ethics Committee for Animal Research of Nanjing University. After the mice had acclimated for 2 weeks, $2 \times 10^{6}$ BGC823-MK cells in $0.1 \mathrm{~mL}$ PBS were injected subcutaneously into the flanks. Mice were monitored for solid tumor growth for $\sim 10$ days, and when the tumor volume reached $50 \mathrm{~mm}^{3}, 20$ mice were randomly assigned to 4 groups $(\mathrm{N}=5)$. Mice then received intravenous injections every other day for 2 weeks as follows: group 1, PBS; group 2, scFv (5.47 mg/kg); group 3 , scFv + DOX (5.47 mg/kg scFv + $2 \mathrm{mg} / \mathrm{kg}$ DOX); group 4, scFv-DOX (7 mg/kg; equivalent dose scFv and DOX). All injections were made in a $0.1-\mathrm{mL}$ volume.

The tumor dimensions were measured every second day during treatment. After the treatment regimen was complete, the mice were sacrificed, and the tumors were removed, photographed, weighed, and prepared for histological analysis. The tumor volume, in $\mathrm{mm}^{3}$, was calculated as volume $=(\text { width })^{2} \times(0.5 \times$ length $)$. The inhibition of tumor growth was calculated as \% inhibition $=(1$ - [average tumor weight for treatment group / average tumor weight for the control group]) $\times 100 \%$.

\section{Histological analysis of tumor cells and tissues}

Excised tumors were gently pressed through a nylon mesh into cold PBS to prepare single-cell suspensions. Cells were centrifuged, washed, and then fixed by resuspension in $1 \%$ paraformaldehyde solution, and were then immediately analyzed by flow cytometry (Becton-Dickinson, USA) (27) and fluorescence microscopy (Carl Zeiss, Germany). The TUNEL (Boster, China) assay for detecting apoptosis in solid tumors was used to compare the cytotoxicity of each drug group.

\section{Statistical analysis}

Data are reported as means $\pm S D$ and were analyzed statistically by one-way analysis of variance (ANOVA) followed by the post hoc Bonferroni test, using the GraphPad Prism 5.0 software. $P<0.05$ was considered to be statistically significant.

\section{Results}

\section{Purification of the ScFv}

SDS-PAGE analysis of the lysates from transfected BL21 cells showed that the cells expressed a 30-kDa scFv protein (Figure 1A) in inclusion bodies. After denaturation and renaturation of inclusion body proteins, scFv was purified by metal affinity chromatography using an Ni-NTA resin. The column specifically bound the His-tag, which was placed at the C-terminal of scFv. SDS-PAGE analysis indicated that the scFv proteins were relatively homogeneous (Figure 1A).

\section{Characterization of immunoconjugates}

We used the exposed aldehyde groups of PAD as a bridge to link the amino groups of DOX with the scFv through the formation of a Schiff bond. Sodium borohydride was used to ensure the stability of the Schiff bond. Excess sodium borohydride reduced the cytotoxicity of DOX (data not shown). The reduction capacity of sodium borohydride as used here was one-third the oxidation capacity of $\mathrm{NalO}_{4}$.

After conjugation, scFv-DOX was purified on a Sephadex 
G150 column (Figure 1B). Protein elution was monitored by absorbance at $280 \mathrm{~nm}$, and the first eluted peak was collected. SDS-PAGE analysis showed that this peak contained scFv-DOX conjugates (Figure 1A). Because Dex and DOX included in scFv-DOX do not represent the protein of interest,
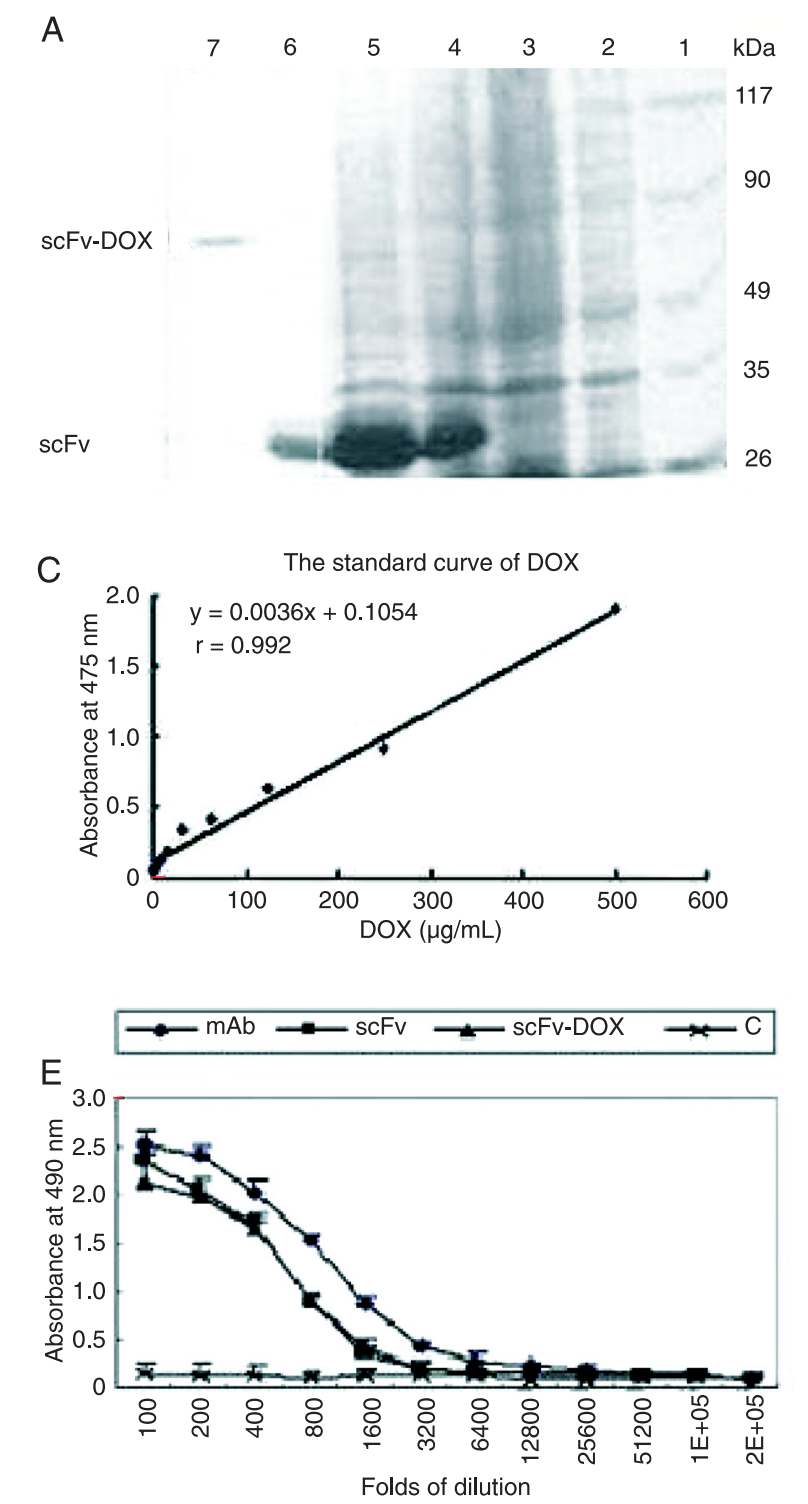

the molecular mass of the conjugate is not $90 \mathrm{kDa}$, as shown in Figure 1. Impurities were removed by passing the solution through a $0.22-\mu \mathrm{m}$ filter. Analysis of the protein, DOX, and Dex content of the conjugate showed the concentrations of scFv, DOX, and Dex to be $502,248.5$, and $216.68 \mu \mathrm{g} / \mathrm{mL}$, respectively
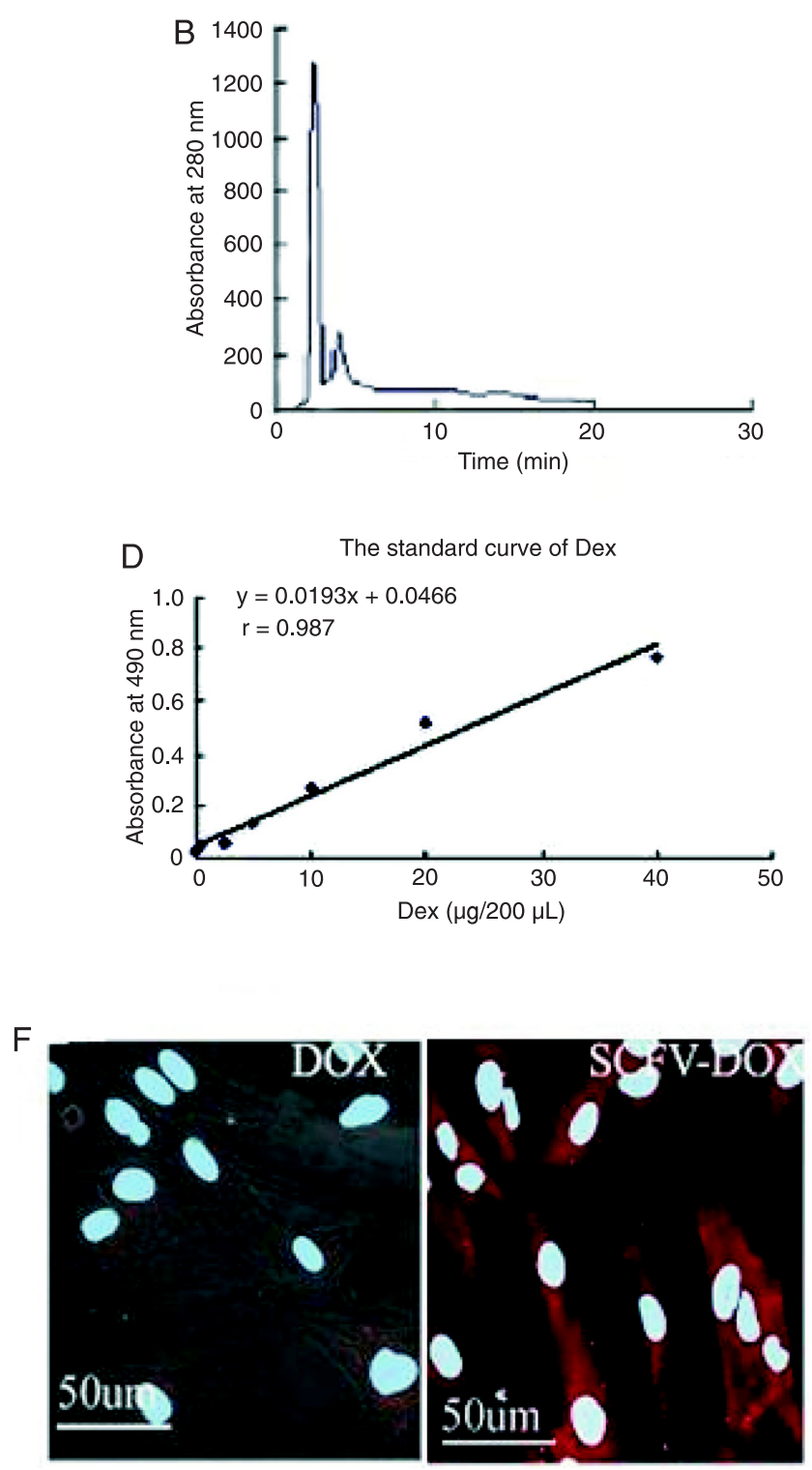

Figure 1. Analysis of doxorubicin (DOX) and single-chain variable fragment (scFv) immunoconjugates. $A$, SDS-PAGE analyses of the expression of scFv and scFv-DOX. Lane 1 = Markers; lanes 2 and $3=$ whole-cell extracts $(20 \mathrm{mg} / \mathrm{lane})$ of scFv vector-transformed Escherichia coli; lane 4 = whole-cell extracts of scFv vector-transformed $E$. coli after induction with isopropyl-1-thio- $\beta$-D-galactopyranoside (IPTG) for $4 \mathrm{~h}$ at $37^{\circ} \mathrm{C}$; lane $5=$ protein from inclusion bodies; lane $6=$ purified scFv; lane $7=$ purified scFv-DOX. B, The absorbance of 280 $\mathrm{nm}$ curve of scFv-DOX conjugates. The first peak was collected and shown by SDS-PAGE to contain the scFv-DOX conjugate. $C$ and $D$, Standard curves for DOX and dextran (Dex), showing that the absorbances of 475 and $490 \mathrm{~nm}$ for scFv-DOX were in the linear range of the assays; 0.7892 and 0.883 , respectively. E, ELISA comparing the affinities of the parental mAb, scFv, scFv-DOX, and an irrelevant antibody $(C)$ as a control. Points are means of triplicates. The results showed that the scFv constructs had the same affinity as the original mAb. $F$, Fluorescence tests on human mesenchymal stem cells (MSC). Blue = nucleus; red = midkine (MK) expressed in the MSC. Left = cells incubated with DOX; right = cells incubated with scFv-DOX. Images show that scFv-DOX bound to the endogenous MK. 


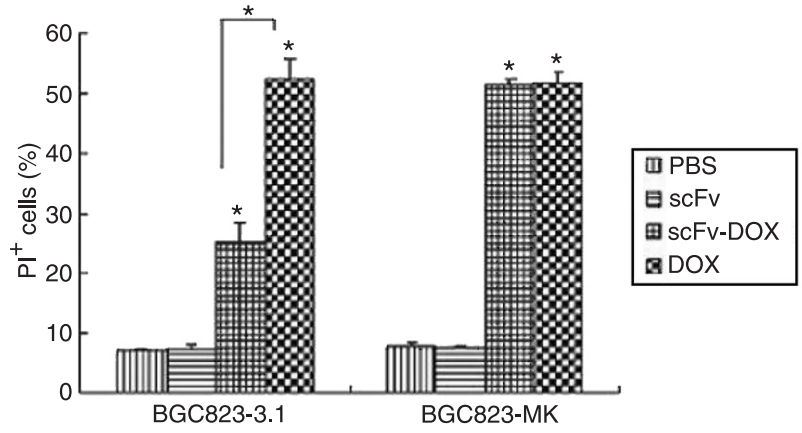

Figure 2. Cytotoxic effects of scFv, scFv-DOX, and DOX on BGC823 and BGC823-MK cells, measured by double staining with propidium iodide $(\mathrm{PI})$. The graph shows the percentages of $\mathrm{PI}^{+}$(apoptotic) cells as the means \pm SEM for three independent experiments. $\mathrm{PBS}=$ phosphate-buffered saline; $\mathrm{scFv}=$ singlechain variable fragment; $\mathrm{DOX}=$ doxorubicin; $\mathrm{MK}=$ midkine . ${ }^{*} \mathrm{P}<0.05$ versus scFv-DOX group (one-way ANOVA followed by the Tukey-Kramer multiple-comparison test).

(Figure 1C and D). This translates to an scFv:Dex:DOX ratio of $1: 1.3: 20$ in the conjugate, with 1 molecule of scFv carrying 20 molecules of DOX. The antigen-binding characteristics of the immunoconjugate were analyzed by ELISA. The results shown in Figure 1E indicate that the scFv-DOX immunoconjugate exhibited the same affinity as the original $\mathrm{mAb}$ and the nonconjugated homologous scFv. Finally, immunofluorescence imaging of stained MSC showed that the ScFv-DOX conjugate retained the ability to bind endogenous MK (Figure 1F).

\section{In vitro cytotoxicity of ScFv-DOX to cells overexpressing MK}

The cytotoxic effect of scFv-DOX was compared with that of free scFv and DOX with untransfected and MK-transfected BGC823 cells. To this end, apoptosis was analyzed by PI staining (Figure 2). The results showed that scFv-DOX cytotoxicity to untransfected BGC823 cells (22.4 $\pm 3.8 \%$ apoptotic cells) was much lower than that of similar amounts $(2.5 \mu \mathrm{g} / \mathrm{mL})$ of free DOX (52.3 $\pm 4.3 \%)$. However, both scFv-DOX and free DOX exhibited similar cytotoxicity (55.3\%) towards BGC823-MK cells. This suggested that conjugating DOX to the MK-specific scFv had reduced DOX toxicity to normal cells expressing low levels of MK, as compared to the toxicity observed for tumor cells overexpressing MK. Therefore, conjugation to scFv enhanced the antigen-specific toxicity of DOX. Consistent with this finding, addition of scFv alone had no cytotoxic effect on the untransfected or transfected cells.

scFv-DOX inhibits BGC823-MK tumor growth in nude mice

Experiments were performed in vivo to test the effects of the scFv-DOX conjugates in mice with established BGC823-MK tumors. The injections of PBS, scFv, DOX + scFv, and scFvDOX conjugates (containing equivalent amounts of ScFv and DOX) were initiated 10 days after tumor implantation, when the tumors were approximately $50 \mathrm{~mm}^{3}$ in volume. Five mice in the PBS and ScFv groups developed tumor masses of similar

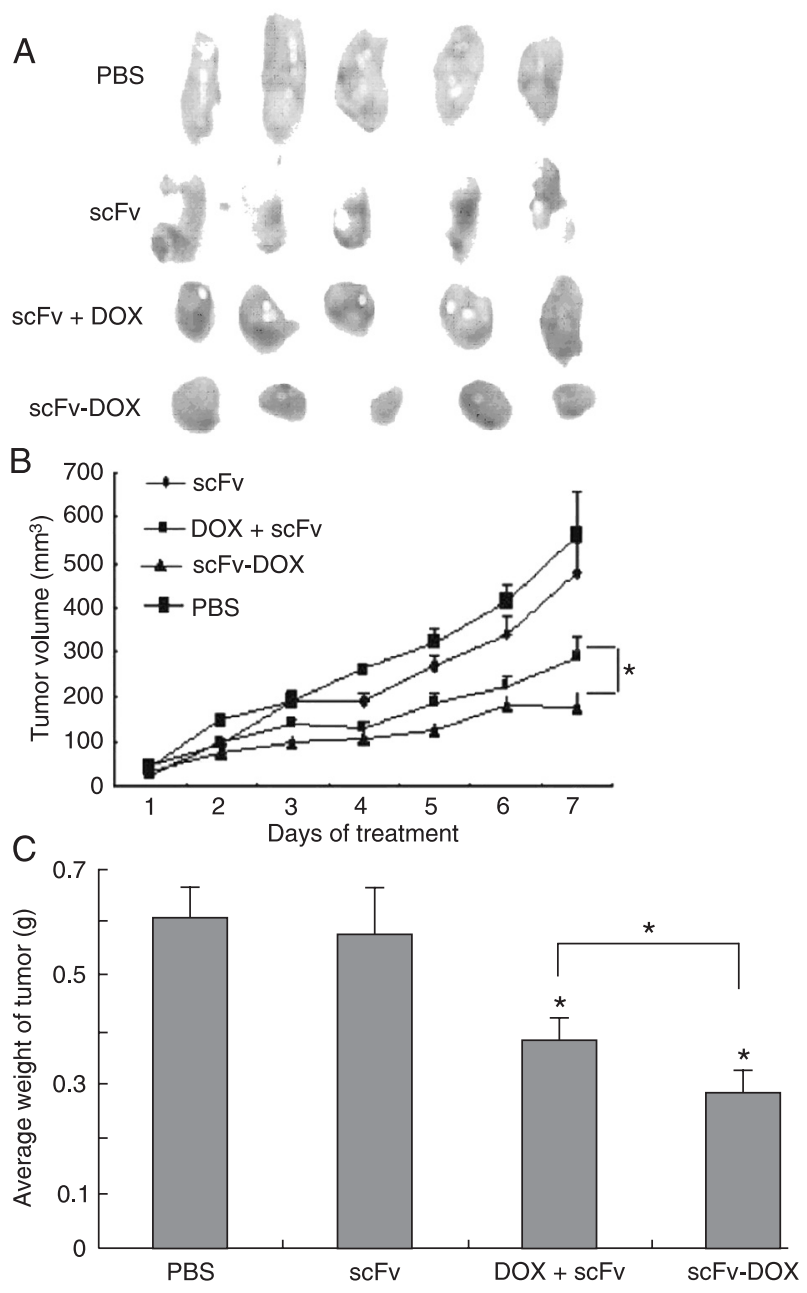

Figure 3. Antitumor activity of scFv-DOX conjugates on subcutaneous solid BGC823-MK tumors in nude mice. A, Photograph showing tumors of animals from the four treatment groups. $B$, Tumor growth during treatment. $C$, Average tumor weights. The average weight of tumors treated with free DOX + scFv or scFvDOX was less than that observed in the control and ScFv-treated groups. Average tumor weight was significantly different between the DOX + scFv and scFv-DOX groups. PBS = phosphate-buffered saline; scFv-DOX = single-chain variable fragment-doxorubicin. ${ }^{*} P<0.05$ versus control (one-way ANOVA followed by the Tukey-Kramer multiple-comparison test).

size (560.34 and $492.85 \mathrm{~mm}^{3}$, respectively) and weight (0.601 and $0.581 \mathrm{~g}$, respectively). In contrast, a significant delay in tumor development was observed in the 5 mice that received $\mathrm{DOX}+\mathrm{scFv}$ or scFv-DOX conjugate treatment. The average tumor volume $\left(180.03 \mathrm{~mm}^{3}\right)$ and average weight $(0.254 \mathrm{~g})$ in the scFv-DOX group were less than in the DOX + scFv group (269.4 $\mathrm{mm}^{3}$ and $0.356 \mathrm{~g}$; Figure 3 ). Thus, the antitumor effect of scFv-DOX was stronger than that exerted by the equivalent amounts of free scFv + free DOX, resulting in inhibition of tumor growth by $51.83(P<0.05)$ and $40.81 \%$ $(P<0.05)$, respectively. 
To determine whether ScFv could promote the accumulation of DOX in the tumors and contribute to apoptosis, single-cell suspensions were prepared from tumors. Flow cytometry analysis of the cells suggested that scFv-DOX accumulation was markedly enhanced compared with tumor cells from animals treated with DOX + scFv (Figure 4A). The geometric mean fluorescence intensity of scFv-specific fluorescence ranged from 9.12 (the scFv + DOX group) to
23.1 (the scFv-DOX group; Figure 4B). These results were confirmed by the results of MSC fluorescence microscopy (Figure 4C). Finally, the number of apoptotic cells in the tumors from animals treated with scFv-DOX was markedly increased compared to the tumors from animals treated with scFv + DOX (Figure 4D). Collectively, these results indicate that scFv-DOX represents a potential therapy to target tumors.

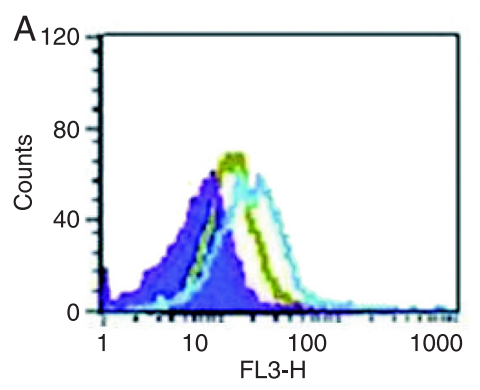

C
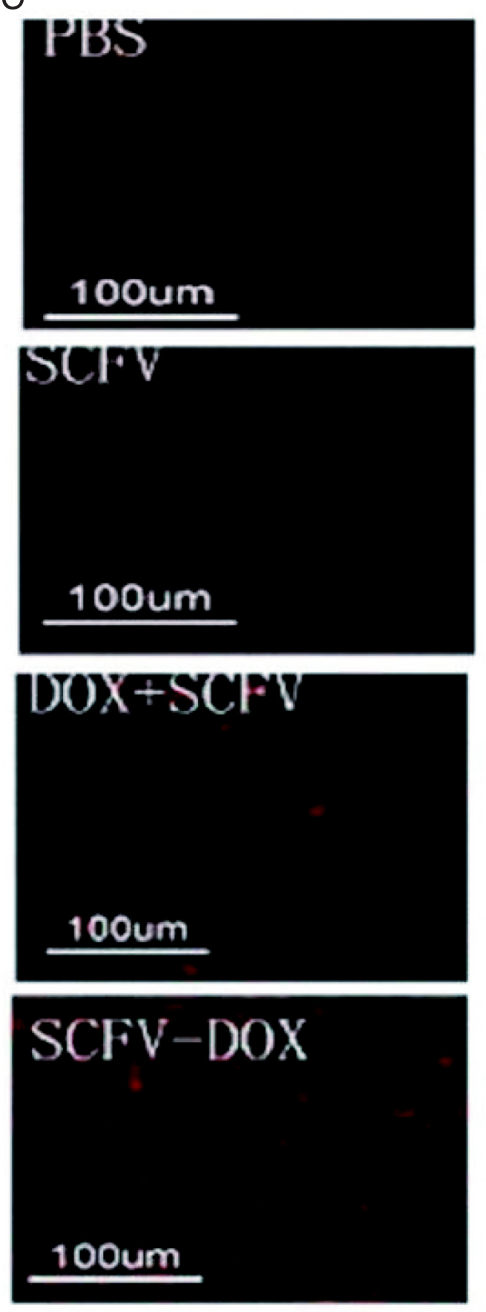

B

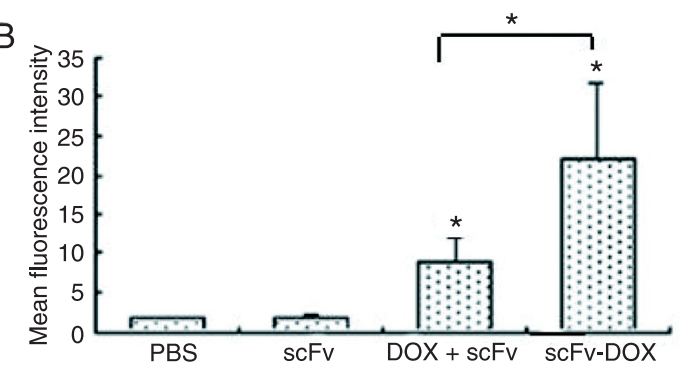

D
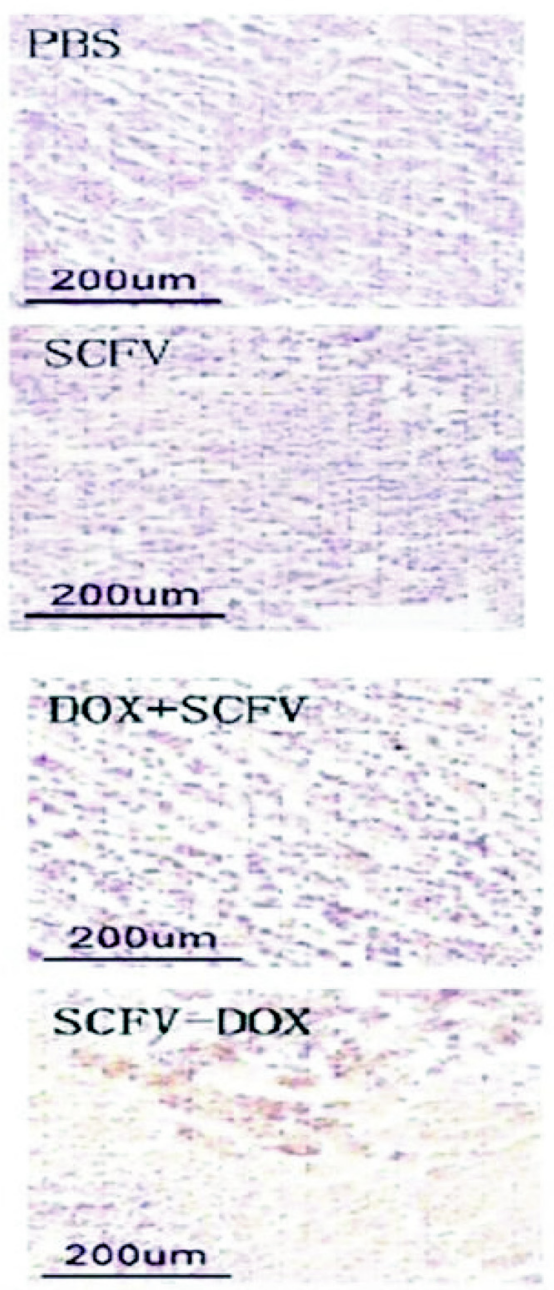

Figure 4. Histological analysis of tumor tissues. $A$ and $B$, Flow cytometry analysis of single-cell suspensions of tumor tissue. A, Purple: PBS group, red: scFv group, green: DOX + scFv group, blue: scFvDOX group. The figure is from a representative experiment. $B$, Histogram of data shown in A. Data are reported as means \pm SEM. PBS = phosphate-buffered saline; $\mathrm{scFv}=$ single-chain variable fragment; $\mathrm{DOX}=$ doxorubicin. ${ }^{*} \mathrm{P}<0.05$ compared to the DOX + scFv group (one-way ANOVA followed by the Tukey-Kramer multiple-comparison test). C, Fluorescence microscopy of single-cell suspensions of tumor tissue. $D$, Apoptosis in the tumor tissue as revealed by the TUNEL apoptosis detection kit. 


\section{Discussion}

The results of a previous study suggested that MK could act as a cancer biomarker and a target for anti-cancer therapy $(10,27)$. To pursue this line of research, we produced the 9E4 hybridoma, which secretes a high-affinity mAb against MK. However, subsequent studies showed that this $m A b$ was not neutralizing and did not inhibit the growth of BGC823-MK cells. Because full-length mAbs have intrinsic shortcomings as drug carriers, we prepared scFv-DOX conjugates of the MK-specific mAb and explored the feasibility of using them as a means to target this anticancer drug to MK-expressing tumor cells.

Conjugation of DOX to polyclonal antibodies or to mAbs through a Dex bridge has been reported $(22,23,28)$. We were the first to use a DOX-scFv conjugate connected through a Dex bridge as a targeted anti-cancer drug. In the preparation of the scFv-DOX conjugate, we used 20,10 , and $5 \mathrm{mg} \mathrm{scFv}$ to conjugate to DOX. The ELISA experiments showed that immunoconjugates formed with either 20 or $10 \mathrm{mg} \mathrm{scFv}$ retained the same binding activity as non-conjugated homologous scFv. However, the combination with $5 \mathrm{mg} \mathrm{scFv}$ was not effective, because more connected DOX decreased the affinity of this group of scFv. The absorbance at $475 \mathrm{~nm}$ showed that each scFv molecule of the 10-mg group had conjugated to $30 \%$ more DOX molecules than had the conjugates with scFv of the 20-mg group (data not shown). Therefore, we selected the immune complexes formed with $10 \mathrm{mg} \mathrm{scFv}$ for future experiments. This quantity of scFv not only had the same affinity as the parent anti-MK mAb, but also bound more DOX (1:20).

The conjugation of Dex to a drug or antibody requires free aldehyde groups available on the oxidized spacer. These aldehyde groups then bind to functional free amino groups on the drug or antibodies by forming Schiff bases. To ensure the stability of these complexes, the excess aldehyde present after conjugation was reduced with sodium borohydride. Because the Dex is an inert, soluble, and nontoxic volume expander in humans $(28,29)$, we did

\section{References}

1. Yoong J, Michael M, Leong T. Targeted therapies for gastric cancer: current status. Drugs 2011; 71: 1367-1384.

2. Muramatsu T. Midkine and pleiotrophin: two related proteins involved in development, survival, inflammation and tumorigenesis. J Biochem 2002; 132: 359-371.

3. Muramatsu T. Midkine. In: Anonymous, Wiley encyclopedia of molecular medicine. New York: John Wiley \& Sons; 2002. p 2086-2088.

4. Kadomatsu K, Tomomura M, Muramatsu T. cDNA cloning and sequencing of a new gene intensely expressed in early differentiation stages of embryonal carcinoma cells and in mid-gestation period of mouse embryogenesis. Biochem Biophys Res Commun 1988; 151: 1312-1318. not include a Dex-DOX conjugate control group for the experiments presented here.

The purified immunoconjugates were used for several in vitro experiments, and for an in vivo experiment with BGC823-MK tumors formed in nude mice. In other applications in which DOX was a component of targeted anti-cancer therapies, DOX was conjugated to antibodies and peptides by the active succinate and the direct carbodiimide methods (30). However, the products of these approaches exhibited reduced cytotoxic activity (31). In the present study, in vitro cell experiments showed that scFv-DOX cytotoxicity (22.4\%) against BGC823 cells expressing low levels of MK was much lower than that exerted by the combination of free DOX + scFv (52.3\%). However, these constructs exerted similar cytotoxicity (55.3\%) against the MK-transfected BGC823 cells, indicating that conjugation of DOX directly with the scFv could reduce DOX toxicity to normal cells expressing low levels of MK, but did not compromise its ability to kill tumor cells overexpressing MK.

For the experiments with the in vivo BGC823-MK cell tumor model, the tumor tissues were analyzed by flow cytometry and fluorescence microscopy. The results showed that scFv could enhance the accumulation of DOX drugs in the tumor cells and tissues to inhibit tumor growth.

In the present study, we did not address two important aspects of our drug conjugates, namely cardiac toxicity and immunogenicity. Nevertheless, we explored scFv as a potential carrier molecule and demonstrated that the cytotoxic drug DOX could be conjugated to an ScFv by Dex, and that the resulting conjugate has potential as a targeted anticancer drug.

\section{Acknowledgments}

Research supported by the National Natural Science Foundation of China (Grant \#30872941) and the scientific and technological major special project "Major Creation of New Drugs" (\#2009ZX09102-205).

5. Dai LC. Midkine translocated to nucleoli and involved in carcinogenesis. World J Gastroenterol 2009; 15: 412-416.

6. Huang $\mathrm{Y}$, Cao G, Wang H, Wang Q, Hou Y. The expression and location of midkine in gastric carcinomas of Chinese patients. Cell Mol Immunol 2007; 4: 135-140.

7. Wang QL, Wang $\mathrm{H}$, Zhao SL, Huang YH, Hou YY. Overexpressed and truncated midkines promote proliferation of BGC823 cells in vitro and tumor growth in vivo. World $J$ Gastroenterol 2008; 14: 1858-1865.

8. Wang Q, Huang $\mathrm{Y}, \mathrm{Ni}$ Y, Wang H, Hou Y. siRNA targeting midkine inhibits gastric cancer cells growth and induces apoptosis involved caspase-3,8,9 activation and mitochondrial depolarization. J Biomed Sci 2007; 14: 783-795. 
9. Ibusuki M, Fujimori H, Yamamoto Y, Ota K, Ueda M, Shinriki $\mathrm{S}$, et al. Midkine in plasma as a novel breast cancer marker. Cancer Sci 2009; 100: 1735-1739.

10. Maehara H, Kaname T, Yanagi K, Hanzawa H, Owan I, Kinjou T, et al. Midkine as a novel target for antibody therapy in osteosarcoma. Biochem Biophys Res Commun 2007; 358: 757-762.

11. Sznol M, Davis T. Tumor antigens as targets for anticancer drug development. Chapter 9. Anticancer Drug Dev 2002; 157-170.

12. Jaracz S, Chen J, Kuznetsova LV, Ojima I. Recent advances in tumor-targeting anticancer drug conjugates. Bioorg Med Chem 2005; 13: 5043-5054.

13. Huston JS, Mudgett-Hunter M, Tai MS, McCartney J, Warren $F$, Haber E, et al. Protein engineering of single-chain Fv analogs and fusion proteins. Methods Enzymol 1991; 203: 46-88.

14. Hudson PJ. Recombinant antibody fragments. Curr Opin Biotechnol 1998; 9: 395-402.

15. Chester KA, Robson L, Keep PA, Pedley RB, Boden JA, Boxer GM, et al. Production and tumour-binding characterization of a chimeric anti-CEA Fab expressed in Escherichia coli. Int J Cancer 1994; 57: 67-72.

16. Schier R, Bye J, Apell G, McCall A, Adams GP, Malmqvist $M$, et al. Isolation of high-affinity monomeric human anti-cerbB-2 single chain Fv using affinity-driven selection. $J \mathrm{Mol}$ Biol 1996; 255: 28-43.

17. Dougan DA, Malby RL, Gruen LC, Kortt AA, Hudson PJ. Effects of substitutions in the binding surface of an antibody on antigen affinity. Protein Eng 1998; 11: 65-74.

18. Sanchez L, Ayala M, Freyre F, Pedroso I, Bell H, Falcon V, et al. High cytoplasmic expression in E. coli, purification, and in vitro refolding of a single chain Fv antibody fragment against the hepatitis B surface antigen. J Biotechnol 1999; 72: $13-20$.

19. Shuli Z, Hui W, Qingling W, Guangfeng Z, Yayi H. Production and characterization of a bacterial single-chain $\mathrm{Fv}$ fragment specific to human midkine. Proceedings of the 5th International Forum on Post-genome Technologies. 2007 Sept. 10-11; Suzhou. p 48-50.

20. Gewirtz DA. A critical evaluation of the mechanisms of action proposed for the antitumor effects of the anthracycline antibiotics adriamycin and daunorubicin. Biochem Pharmacol 1999; 57: 727-741.
21. Dansithong W, Paul S, Mitsumoto T, Saruhashi S, Shinozawa T. Production and characterization of a bacterial single-chain Fv fragment specific to human truncated midkine. Cancer Lett 2001; 164: 169-176.

22. Inoh K, Muramatsu $H$, Torii S, Ikematsu S, Oda M, Kumai $\mathrm{H}$, et al. Doxorubicin-conjugated anti-midkine monoclonal antibody as a potential anti-tumor drug. Jpn J Clin Oncol 2006; 36: 207-211.

23. Daejin K, Talia H, Archana M, Annie W, Wei-Ting H, Sara IP, et al. Delivery of chemotherapeutic agents using drugloaded irradiated tumor cells to treat murine ovarian tumors. J Biomed Sci 2010; 17: 61.

24. Dubois M, Gilles K, Hamilton J, Rebers P, Smith F. Colorimetric method for determination of sugars and related substances. Anal Chem 1956; 28: 350-356.

25. Aboud-Pirak E, Hurwitz E, Bellot F, Schlessinger J, Sela M. Inhibition of human tumor growth in nude mice by a conjugate of doxorubicin with monoclonal antibodies to epidermal growth factor receptor. Proc Natl Acad Sci U S A 1989; 86: 3778-3781.

26. Zhang H, Liu X, Chen S, Wu J, Ye X, Xu L, et al. Tectorigenin inhibits the in vitro proliferation and enhances miR-338* expression of pulmonary fibroblasts in rats with idiopathic pulmonary fibrosis. J Ethnopharmacol 2010; 131: 165-173.

27. Kerzerho J, Adotevi O, Castelli FA, Dosset M, Bernardeau K, Szely N, et al. The angiogenic growth factor and biomarker midkine is a tumor-shared antigen. J Immunol 2010; 185: 418-423.

28. Shouval D, Adler R, Wands JR, Hurwitz E, Isselbacher KJ, Sela M. Doxorubicin conjugates of monoclonal antibodies to hepatoma-associated antigens. Proc Natl Acad Sci U S A 1988; 85: 8276-8280.

29. Rakestraw SL, Tompkins RG, Yarmush ML. Antibodytargeted photolysis: in vitro studies with Sn(IV) chlorin e6 covalently bound to monoclonal antibodies using a modified dextran carrier. Proc Natl Acad Sci U S A 1990; 87: 42174221.

30. Arap W, Pasqualini R, Ruoslahti E. Cancer treatment by targeted drug delivery to tumor vasculature in a mouse model. Science 1998; 279: 377-380.

31. Hurwitz E, Levy R, Maron R, Wilchek M, Arnon R, Sela M. The covalent binding of daunomycin and adriamycin to antibodies, with retention of both drug and antibody activities. Cancer Res 1975; 35: 1175-1181. 